\title{
Positron Emission Tomography and Stage Migration in Head and Neck Cancer
}

Noam A. VanderWalde, MD; Ramzi G. Salloum, PhD; Tsai-Ling Liu, MSPH; Mark C. Hornbrook, PhD; Maureen C. O'Keeffe Rosetti, MS; Debra P. Ritzwoller, PhD; Paul A. Fishman, PhD; Jennifer Elston Lafata, PhD; Amir H. Khandani, MD; Bhishamjit S. Chera, MD

IMPORTANCE Since 2001, there has been a rapid adoption of positron emission tomography (PET) for diagnosis and American Joint Committee on Cancer (AJCC) staging of head and neck cancer (HNC) without data describing improved clinical outcomes.

OBJECTIVE To determine the association between increased use of PET and stage and/or survival for patients with $\mathrm{HNC}$ in the managed care environment.

DESIGN, SETTING, AND PARTICIPANTS Adult patients diagnosed as having HNC $(\mathrm{n}=958)$ from 2000 to 2008 at 4 integrated health systems were identified via tumor registries linked to administrative data. The AJCC stage distribution, patient and treatment characteristics, and survival between pre-PET era (2000-2004) vs PET era (2005-2008) and use of PET vs no use of PET during the PET era were compared. The AJCC stages were categorized to represent localized (stage I or II), locally advanced (stage III, IVA, or IVB), and metastatic (stage IVC) disease.

INTERVENTIONS Treatments were determined by billing codes for surgery, radiation treatment, and chemotherapy.

MAIN OUTCOMES AND MEASURES The primary outcome for this study was the use of PET. Secondary outcomes included treatment received and 2-year survival. A logit model estimated the effects of PET on diagnosis of locally advanced disease. Kaplan-Meier estimates described overall survival differences between PET and non-PET. Cox regression evaluated the association of PET on survival in patients with locally advanced disease.

RESULTS An association between PET and locally advanced disease was found (odds ratio, 2.86 [95\% Cl, 1.90-4.29) $(P<.001)$. Two-year overall survival for patients with locally advanced disease with and without PET was $52 \%$ and $32 \%$, respectively $(P=.004)$, but there was no difference for all stages $(P=.69)$. On Cox proportional hazard regression, $\mathrm{PET}$ had no association with survival in patients with locally advanced disease (hazard ratio, 1.208 [95\% $\mathrm{Cl}, 0.778-1.877])(P=.40)$.

CONCLUSIONS AND RELEVANCE The increasing use of PET among patients with $\mathrm{HNC}$ is associated with a greater number of patients with higher-stage disease and a dilution of the population with higher-stage disease with patients who have a better prognosis. Thus, the improved survival in patients with locally advanced disease likely reflects selection bias and stage migration. Further research on PET use among patients with $\mathrm{HNC}$ is necessary to determine if it results in improved treatment for individual patients.

JAMA Otolaryngol Head Neck Surg. 2014;140(7):654-661. doi:10.1001/jamaoto.2014.812 Published online May 29, 2014.

Author Affiliations: Author affiliations are listed at the end of this article.

Corresponding Author: Bhishamjit S. Chera, MD, Department of Radiation Oncology, University of North Carolina Hospitals, 101 Manning Dr، CB \#7512, Chapel Hill, NC 27514 (bchera@med.unc.edu). 
n 2001 the Centers for Medicare and Medicaid Services (CMS) began to cover fluorodeoxyglucose (FDG) positron emission tomography (PET) for diagnosing, staging, and restaging of head and neck cancer (HNC). Multiple studies have demonstrated the increased sensitivity of FDG PET over computed tomography (CT) for detecting primary tumors, regional nodal disease, and distant metastases. ${ }^{1-7}$ This improvement in diagnostic staging often results in changes in therapeutic recommendations for surgical and radiation treatment as well as changes to radiation treatment fields. ${ }^{8-11} \mathrm{Be}-$ cause of potential treatment changes and prognostic information, as well as patient and physician preference, FDG PET has been rapidly adopted for managing HNC. ${ }^{12}$ However, whether these changes result in better clinical outcomes is not entirely known.

Because FDG PET is more sensitive than $\mathrm{CT},{ }^{3}$ it often leads to patients being assigned a higher stage than if they were staged with CT alone. ${ }^{9}$ For example, a recent study reported that $17 \%$ of patients with HNC were assigned a higher stage after undergoing FDG PET compared with CT alone. ${ }^{9}$ The tendency for FDG PET to lead to "up-staging" of HNC may lead to better stagespecific survival: ie, FDG PET-staged patients with higherstage disease having a better prognosis than comparable CT alone-staged patients. This dilution effect is often referred to as the Will Rogers phenomenon, or stage migration. ${ }^{13}$ The concept of stage migration was first described by Feinstein and colleagues $^{13}$ in a cohort of patients with lung cancer. The researchers found that patients who had been diagnosed using CT, radionuclide scanning, and ultrasonography had higher 6-month survival and stage-specific survival than those who were diagnosed based on "old" diagnostic tools. However, they demonstrated that if all of the patients were staged based on clinical symptoms that were unaltered by new diagnostic techniques, the 2 cohorts had similar survival periods. Thus, they hypothesized that use of the new technologies caused a migration of better-prognosis patients into higher stages of disease, leading to the appearance of improved survival length in both lower- and higher-staged patients rather than improvement in individual patient care. They named this the Will Rogers phenomenon after the humorist who reportedly once joked about a geographic migration that occurred during the Great Depression: "When the Okies left Oklahoma and moved to California, they raised the average intelligence level in both states." While the quote attribution to Will Rogers cannot be documented, the statistical concept it articulates is solid.

The hypothesis of the present study is that the increased use of FDG PET over time is associated with the appearance of improved stage-specific survival due to a stage migration phenomenon. To test this hypothesis, we analyzed the use of FDG PET in a large multisite sample of managed care patients in the United States.

\section{Methods}

Study Population and Setting

The study involved only retrospective administrative data review, so patient informed consent was waived.
This analysis was conducted within 4 large, nonprofit, integrated health systems: Group Health Cooperative (Seattle, Washington), Health Alliance Plan-Henry Ford Health System (Detroit, Michigan), Kaiser Permanente Colorado (Denver), and Kaiser Permanente Northwest (Portland, Oregon). Institutional review boards at each of the 4 participating health systems approved all aspects of the study protocol. These plans are all members of the Cancer Research Network (National Cancer Institute [NCI] Cooperative Agreement No. U19 CA79689, Increasing Effectiveness of Cancer Control Interventions), an initiative of the NCI designed to conduct research on cancer prevention, early detection, treatment, long-term care, surveillance, and cancer communication dissemination and implementation research.

Data available from each health system's tumor registry were used to identify patients older than 18 years who were diagnosed as having HNC between 2000 and 2008. We classified patients by tumor site as follows: oral cavity (International Classification for Diseases for Oncology [ICD-O] codes Cooo-Coog and C019-C069), oropharynx (ICD-O codes Co90-C099 and C100-C109), nasopharynx (ICD-O codes C110-C119), larynx and/or hypopharynx (ICD-O codes C129C139 and C320-C329), salivary gland (ICD-O codes Co79C089), and sinuses and/or pharynx unspecified (ICD-O codes C140, C142, and C148). Patients eligible for study inclusion were those continuously enrolled in the health plan for the 12-month period preceding their date of cancer diagnosis. Patients whose stage of disease at the time of diagnosis was not available were excluded. Patients were also excluded if they had a previous diagnosis of invasive cancer. For HNC diagnosis and staging, sample patients were observed from an index date, defined as 60 days preceding their HNC diagnosis date, until the earliest of the following end points: death, health plan disenrollment, 5 years after the initial cancer diagnosis, or the end of the follow-up period (December 31, 2008). Comorbidities were identified from a period of 12 months prior to diagnosis.

\section{Data Sources and Measures}

Automated electronic medical record (EMR) data linked to tumor registry data were accessed to obtain tumor site, age at diagnosis, sex, date of cancer diagnosis, stage at diagnosis, and comorbidities within the 12-month period preceding diagnosis for each patient. We defined the disease stages into 3 clinically relevant groups: localized (American Joint Committee on Cancer [AJCC] stages I and II), locally advanced (stages III, IVA, and IVB), and metastatic (stage IVC). Administrative data and EMRs were reviewed between the patient's index date and end date to compute receipt of imaging services and treatment with surgery, chemotherapy, and radiation. Receipt of imaging tests was ascertained through Current Procedural Terminology (CPT) codes 78111 through 78116 for PET and CPT codes 70450, 70460, 70470, 70486, 70490 through 70492, 71250, 71260, and 71270 for CT. Receipt of treatment type was determined by billing codes and timing of treatment within 120 days following diagnosis. The Deyo adaptation of the Charlson 
comorbidity index was constructed using inpatient and outpatient diagnostic information available in the 12-month period preceding diagnosis. ${ }^{14}$ Because FDG PET was not used in these organizations before $2005,{ }^{15}$ we defined the PET era as the period from 2005 through 2008 and the prePET era as 2000 through 2004. For each patient, we defined the initial phase to include the staging and active treatment periods, beginning 30 days before diagnosis and ending 60 days following the first diagnosis. These dates were chosen based on variability of diagnosis date compared with first treatment as well as timing between surgery (which was often the same as diagnosis date) and first radiation or chemotherapy treatments. Patients who underwent their first PET after their first radiation or chemotherapy treatments and those who underwent first PETs after 60 days from diagnosis were considered as part of a surveillance group, and for the purpose of this study were considered in the no-PET group for staging.

\section{Analytical Approach}

The primary end point in this study was the use of FDG PET among patients with HNC. To establish whether diagnosis era had an effect on staging and survival, a $\chi^{2}$ test or Wilcoxon rank sum test (age, comorbidity, and stage) was used to examine the significance of differences in the distributions of various characteristics between patients with HNC in the pre-PET vs PET eras. For survival analysis comparison, 2-year product-limit survival estimates via KaplanMeier methods were used. Because the PET-era patients had significantly less follow-up time, the survival comparison analysis performed for the PET era vs pre-PET era was limited to patients in the PET era diagnosed in 2005 and 2006 and having at least 2 years of possible follow-up. A separate analysis was performed to examine the significance of differences in the distributions of various characteristics between those who underwent PET and those who did not within the PET era. For these analyses, data from patients diagnosed from 2005 through 2008 were used. Because of the differing lengths of follow-up among sample members, we used the Kaplan-Meier method for estimating overall survival by stage and FDG PET status (ie, with and without) during the PET era. We used a Cox proportional hazards model, which also accounts for the differing length of follow-up among sample members, to quantify the effects of FDG PET use among patients with locally advanced disease, controlling for baseline clinical and sociodemographic patient characteristics, on the risk of death in the PET era. The control variables in the Cox Model included the patient's tumor site, age at diagnosis, sex, Charlson-Deyo comorbidity score, and treatment type. We used a logit model to estimate the association between PET use and diagnosis of locally advanced disease. For this model, we pooled patients in both the pre-PET and PET eras. We controlled for PET use, tumor site, age at diagnosis, sex, and health plan. Analyses were performed using SAS software, version 9.3 (SAS Institute Inc) and STATA software, version 11.0 (StataCorp LP). Statistical results of $P<.01$ were considered to be statistically significant.

\section{Results}

\section{Patient and Treatment Characteristics}

A total of 958 patients identified in this cohort met the study inclusion criteria. Forty-six percent were treated in the PET era. Median patient age was 66 years. Median follow-up for all patients was 31 months (range, 0.1-109.0 months). The median follow-up for patients in the pre-PET era was 56 months. For surviving patients in the PET era, the median follow-up for those who underwent PET was 22 months, and the median follow-up for those who did not undergo PET was 27 months (see eFigure 1 in the Supplement). Most patients were men and had a Charlson comorbidity score of 0 . Oral cavity was the most common primary tumor site, and most patients were diagnosed as having localized disease.

\section{Pre-PET Era vs PET Era}

Bivariate analyses found no statistically significant differences in the primary tumor sites, stage, age, sex, or comorbidities between patients in the pre-PET era and the PET era (Table 1). A significant difference in the type of treatments patients received was detected: less use of surgery (pre-PET era, $66.9 \%$ vs PET era, 53.7\%) $(P<.001)$ and a trend toward increasing use of chemotherapy during the PET era (pre-PET era, 20.5\% vs PET era, $25.9 \%)(P=.05)$. No difference in 2-year overall survival (OS) rates was found (pre-PET era, $75.5 \%$ vs PET era, 74.0\%) $(P=.34)$.

\section{PET vs No PET During the PET Era}

In the PET era, tumor site was significantly associated with use of PET for staging $(P<.001)$ (Table 1$)$. Younger patients and those with later years of diagnosis were more likely to have undergone FDG PET scans $(P<$.001). Use of FDG PET for staging purposes has been increasing since 2005 among this cohort (eFigure 2 in the Supplement). In 2005, about $12.5 \%$ of patients received pretreatment FDG PET scans. By 2008, the percentage increased to $34 \%$. Compared with patients who did not undergo PET, those who underwent PET were more likely to receive radiation treatment (no PET, 48.3\% vs PET, 83.3\% $(P<.001)$, more likely to receive chemotherapy (no PET, $17.5 \%$ vs PET, $43.1 \%)(P<.001)$ and less likely to receive no treatment (no PET, $18.8 \%$ vs PET, 0\%) $(P<.001)$.

\section{PET Association With Staging}

Within the PET era cohort, a statistically significant increase in the number of patients staged as having locally advanced disease was observed (Table 1 and Figure 1). In oral cavity and larynx and/or hypopharynx disease, most patients who underwent PET scans were staged as having locally advanced disease, while those without PET were mostly staged as having local disease (eFigures 3 and 4 in the Supplement). However, this was not the case with oropharynx and nasopharynx (eFigures 5 and 6 in the Supplement). In our logit model of clinically important variables, PET scan use was associated with higher stage of disease. In addition, oropharyngeal cancers were more likely to be assigned a higher stage than oral cavity cancers (Table 2). 
Table 1. Characteristics of All Participants Diagnosed as Having HNC

\begin{tabular}{|c|c|c|c|c|c|c|}
\hline \multirow[b]{2}{*}{ Characteristic } & \multicolumn{2}{|c|}{ Patients, No. (\%) ${ }^{\mathrm{a}}$} & \multirow[b]{2}{*}{$P$ Value } & \multicolumn{2}{|c|}{ Patients in PET Era, No. (\%) } & \multirow[b]{2}{*}{$P$ Value } \\
\hline & Pre-PET Era & PET Era & & No PET Used & PET Used & \\
\hline Total & $522(54.4)$ & $436(45.6)$ & NA & $292(67.0)$ & $144(33.0)$ & NA \\
\hline \multicolumn{7}{|l|}{ Tumor site } \\
\hline Oral cavity & $246(47.1)$ & $186(42.7)$ & \multirow{6}{*}{.49} & $134(45.0)$ & $52(36.1)$ & \multirow{6}{*}{$<.001$} \\
\hline Oropharynx & $66(12.6)$ & $64(14.7)$ & & $22(7.5)$ & $42(29.2)$ & \\
\hline Nasopharynx & $21(4.0)$ & $20(4.6)$ & & $10(3.4)$ & $10(6.9)$ & \\
\hline Larynx and/or hypopharynx & $125(24.0)$ & $97(22.3)$ & & $74(25.3)$ & $23(16.0)$ & \\
\hline Salivary gland & $47(9.0)$ & $50(11.5)$ & & $40(13.7)$ & $10(6.9)$ & \\
\hline $\begin{array}{l}\text { Sinuses and/or pharynx } \\
\text { unspecified }\end{array}$ & $17(3.3)$ & $19(4.4)$ & & $12(4.1)$ & $7(4.9)$ & \\
\hline \multicolumn{7}{|l|}{ Age at diagnosis, $\mathrm{y}^{\mathrm{b}}$} \\
\hline $18-39$ & $13(2.5)$ & $12(2.8)$ & \multirow{5}{*}{.35} & $8(2.7)$ & $4(2.8)$ & \multirow{5}{*}{$<.001$} \\
\hline $40-49$ & $58(11.1)$ & $43(9.9)$ & & $26(8.9)$ & $17(11.8)$ & \\
\hline $50-64$ & $168(32.2)$ & $170(39.0)$ & & $101(34.6)$ & $69(47.9)$ & \\
\hline $65-74$ & $141(27.0)$ & $95(21.8)$ & & $64(21.9)$ & $31(21.5)$ & \\
\hline$\geq 75$ & $142(27.2)$ & $116(26.6)$ & & $93(31.9)$ & $23(16.0)$ & \\
\hline \multicolumn{7}{|l|}{ Sex } \\
\hline Male & $349(66.9)$ & $286(65.6)$ & \multirow{2}{*}{.68} & $188(64.4)$ & $98(68.1)$ & \multirow{2}{*}{.45} \\
\hline Female & $173(33.1)$ & $150(34.4)$ & & $104(35.6)$ & $46(31.9)$ & \\
\hline \multicolumn{7}{|l|}{ Charlson comorbidity score ${ }^{\mathrm{b}}$} \\
\hline 0 & $324(62.1)$ & $256(58.7)$ & \multirow{3}{*}{.19} & $162(55.5)$ & $94(65.3)$ & \multirow{3}{*}{.05} \\
\hline 1 & $117(22.4)$ & $96(22.0)$ & & $67(23.0)$ & $29(20.1)$ & \\
\hline$\geq 2$ & $81(15.5)$ & $84(19.3)$ & & $63(21.6)$ & $21(14.6)$ & \\
\hline \multicolumn{7}{|l|}{ Stage $^{\mathrm{b}}$} \\
\hline I and II & $302(57.9)$ & $227(52.1)$ & \multirow{3}{*}{.06} & $181(62.0)$ & $46(31.9)$ & \multirow{3}{*}{$<.001$} \\
\hline III, IVA, or IVB & $206(39.5)$ & $193(44.3)$ & & $103(35.3)$ & $90(62.5)$ & \\
\hline IVC & $14(2.7)$ & $16(3.7)$ & & $8(2.7)$ & $8(5.6)$ & \\
\hline \multicolumn{7}{|l|}{ Year of diagnosis } \\
\hline 2000 & $84(16.1)$ & NA & & 0 & 0 & \\
\hline 2001 & $90(17.2)$ & NA & & 0 & 0 & \\
\hline 2002 & 97 (18.6) & NA & & 0 & 0 & \\
\hline 2003 & $99(19.0)$ & NA & & 0 & 0 & \\
\hline 2004 & $152(29.1)$ & NA & NA & 0 & 0 & NA \\
\hline 2005 & NA & $110(25.2)$ & & $92(31.5)$ & $18(12.5)$ & \\
\hline 2006 & NA & $109(25.0)$ & & $68(23.3)$ & $41(28.5)$ & \\
\hline 2007 & NA & $119(27.3)$ & & $83(28.4)$ & $36(25.0)$ & \\
\hline 2008 & NA & $98(22.5)$ & & $49(16.8)$ & $49(34.0)$ & \\
\hline Health plan & & & & & & \\
\hline 1 & $44(8.4)$ & $93(21.3)$ & & $63(21.5)$ & $30(20.8)$ & \\
\hline 2 & $114(21.8)$ & 78 (17.9) & < & $68(23.3)$ & $10(6.9)$ & < 01 \\
\hline 3 & $175(33.5)$ & $132(30.3)$ & $<.001$ & $75(25.7)$ & $57(39.6)$ & $<.001$ \\
\hline 4 & $189(36.2)$ & $133(30.5)$ & & $86(29.5)$ & $47(32.6)$ & \\
\hline Treatment & & & & & & \\
\hline Surgery & 349 (66.9) & $234(53.7)$ & $<.001$ & $163(55.8)$ & $71(49.3)$ & .20 \\
\hline Radiotherapy & 323 (61.9) & 261 (59.9) & .52 & $141(48.3)$ & $120(83.3)$ & $<.001$ \\
\hline Chemotherapy & $107(20.5)$ & $113(25.9)$ & .05 & $51(17.5)$ & $62(43.1)$ & $<.001$ \\
\hline No treatment & $55(10.5)$ & $55(12.6)$ & .32 & $55(18.8)$ & 0 & $<.001$ \\
\hline Deaths & $248(47.5)$ & $112(25.7)$ & NA & $75(25.7)$ & $37(25.7)$ & NA \\
\hline 2 -Year survival, \%c & 75.5 & 74.0 & .34 & 55.5 & 53.2 & .69 \\
\hline
\end{tabular}

Abbreviations: NA, not applicable; OR, odds ratio; PET, positron emission tomography.

a The pre-PET era was from 2000 through 2004; PET era, 2005 through 2008.

other variables were assessed with the $\chi^{2}$ test.

${ }^{\mathrm{b}}$ Age, comorbidity, and stage were compared using Wilcoxon-rank sum test; all

c Two-year survival based on product-limit survival estimates. For pre-PET era vs PET era survival comparison, PET era was limited to 2005 and 2006. All other analyses included patients from 2005 through 2008. 
Figure 1. Diagnosis Stage in All Patients Across Eras and PET vs No PET in the PET Era

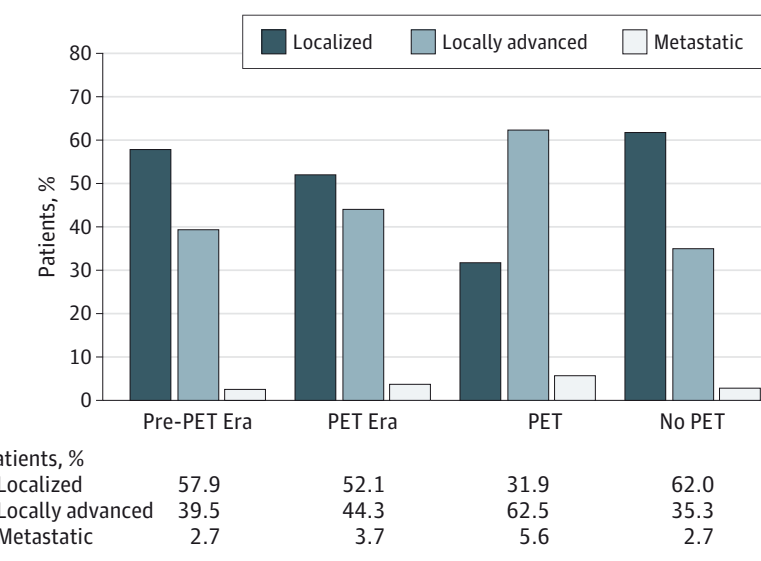

Localized disease was defined as American Joint Committee on Cancer stage I or II; locally advanced, stage III, IVA, or IVB; and metastatic, stage IVC disease. PET indicates positron emission tomography.

PET Association With Survival

Within the PET era, no survival difference was found between those who underwent FDG PET and those who did not (Figure 2A). A significant increase in stage-specific survival was detected for patients with locally advanced disease (Figure 2B). No stage-specific survival differences were found in patients with local disease (Figure 2C) or metastatic disease (not shown). Two-year survival for all patients, no PET vs PET, was 55.5\% vs $53.2 \%(P=.69)$. Two-year survival for locally advanced disease, no PET vs PET, was $32.1 \%$ vs $52.2 \%(P=.004)$. Multivariate Cox regression analysis showed no association between use of PET in locally advanced disease and survival (hazard ratio, 1.208) $(P=.40)$ (Table 3$)$.

\section{Discussion}

In a large multisite cohort of managed care patients, we identified an increasing trend in the use of pretreatment FDG PET scans for patients with HNC since 2005. PET scans were associated with a larger percentage of patients diagnosed as having locally advanced disease and an increase in stage-specific survival for patients with locally advanced disease. However, the use of FDG PET scans was not associated with an overall survival benefit in patients with locally advanced disease on multivariate analysis. Therefore the survival benefit for locally advanced disease is more likely a stage migration phenomenon.

Stage migration, or the Will Rogers phenomenon, ${ }^{13}$ can occur across disease eras, or even in individual patients. A clinical scenario illustrating this phenomenon is as follows: A patient is diagnosed as having stage III salivary gland cancer on the basis of clinical examination and CT. The patient then undergoes a PET-CT scan, which picks up small pulmonary nodules, and the disease is up-staged to IVC. If the patient had not undergone a PET scan, standard stage III treatment would have
Table 2. Logit Model Analysis for the Association of PET Use With Diagnosis of Locally Advanced Disease ${ }^{a}$

\begin{tabular}{llc}
\hline Study Variable & \multicolumn{1}{c}{ OR $(95 \% \mathrm{CI})$} & $P$ Value \\
\hline PET scan vs no PET & $2.858(1.903-4.293)$ & $<.001$ \\
\hline $\begin{array}{l}\text { Age at diagnosis } \\
\text { (continuous) }\end{array}$ & $1.001(0.990-1.011)$ & .92 \\
\hline $\begin{array}{l}\text { Female vs male } \\
\text { Tumor site }\end{array}$ & $0.653(0.484-0.881)$ & .005 \\
\hline $\begin{array}{l}\text { Oral cavity } \\
\text { Oropharynx }\end{array}$ & 1 [Reference] & 1 [Reference] \\
\hline $\begin{array}{l}\text { Nasopharynx } \\
\text { Larynx and/or } \\
\text { hypopharynx }\end{array}$ & $3.338(2.154-5.173)$ & $<.001$ \\
\hline $\begin{array}{l}\text { Salivary gland } \\
\text { Sinuses and/or pharynx } \\
\text { unspecified }\end{array}$ & $1.195(1.067-4.518)$ & .03 \\
\hline \begin{tabular}{l} 
Health plan \\
\hline 1
\end{tabular} & $1.234(0.714(1.142-5.103)$ & .25 \\
\hline 2 & 1 [Reference] & .02 \\
\hline 3 & $1.455(0.901-2.351)$ & 1 [Reference] \\
\hline 4 & $0.709(0.457-1.099)$ & .13 \\
\hline $\begin{array}{l}\text { Abbreviations: OR, odds ratio; PET, positron emission tomography. } \\
\text { a Locally advanced disease is defined as stage III, IVA, or IVB disease }(\mathrm{n}=928), \\
\text { excluding stage IVC. Localized disease (stage I or II) was used as reference. }\end{array}$ \\
\hline
\end{tabular}

been administered, and this would have been recorded as a stage III event; metastatic disease would have been diagnosed months to years later. However, because of the PET findings, the patient migrates into the stage IVC patient population, thus improving the prognosis for the remaining stage III population. This patient will likely live longer than those patients who were diagnosed as having symptomatic distant disease, thus also improving the prognosis for stage IVC patients. The actual survival for both groups has not changed, nor has the survival of the individual patient. More recently, a large SEER-Medicare claims based study ${ }^{16}$ demonstrated a similar phenomenon with increased use of FDG PET scans in non-small-cell lung cancers.

To our knowledge this is the first study to assess FDG PET use and survival outcomes in patients with HNC using a multisite EMR-based data set of both younger and older patients. A single institutional matched retrospective study of $58 \mathrm{pa}-$ tients who received FDG PET staging prior to chemoradiotherapy compared with 58 patients who did not receive FDG PET for staging prior to chemoradiotherapy demonstrated no difference between local control, regional control, distant metastases, cause-specific survival or overall survival. ${ }^{17}$ The results of that study may not be generalizable because of the small sample size, single institution patient population, and retrospective design. Our study also found no difference in overall survival for the whole group but did find a stage migration phenomenon with a survival difference for those with locally advanced disease.

A difference in staging and survival was not identified between the pre-PET and PET eras. This is interesting given the increasing incidence of human papillomavirus (HPV)-related oropharyngeal cancers over this period and their associated higher stages and improved prognoses. ${ }^{18,19}$ Tobacco use and 
Figure 2. Kaplan-Meier Survival Curves Within the PET Era

A Overall survival

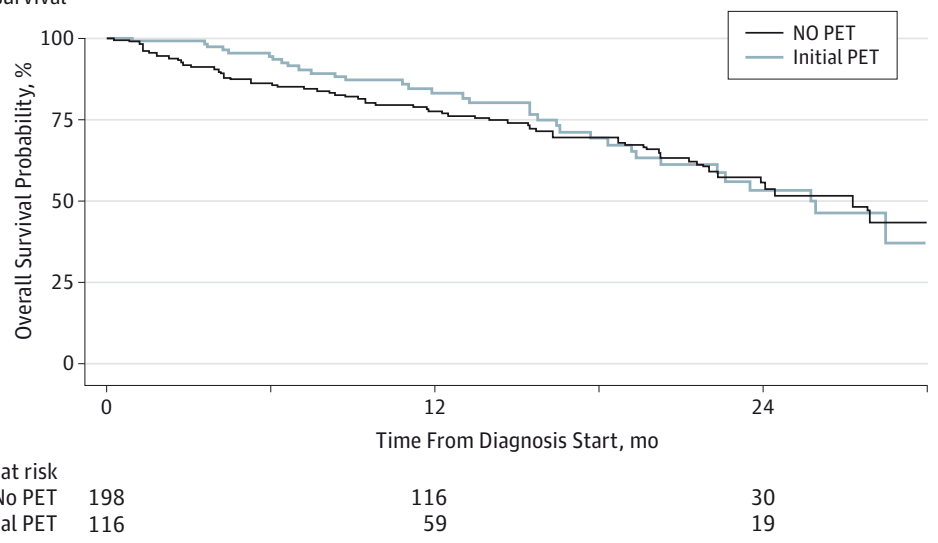

B Stage-specific survival, locally advanced disease

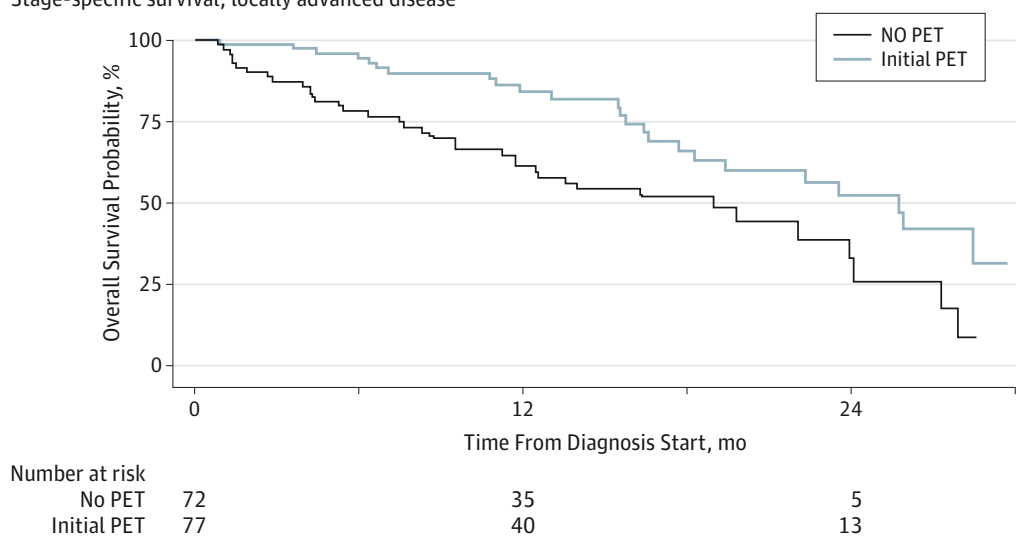

C Stage-specific survival, localized disease

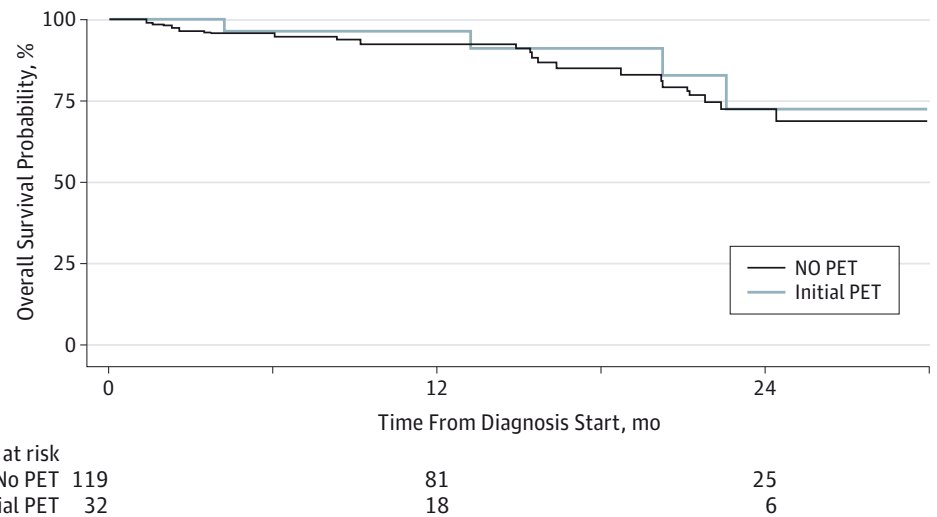

Localized disease was defined as American Joint Committee on Cancer stage I or II; locally advanced, stage III, IVA, or IVB. PET indicates positron emission tomography.

HPV status, 2 important variables, were not available in this cohort, but the lack of improved survival over these eras at least allows us to rule out era as a cause of increased staging or survival. The decrease in surgical treatments across eras found in this study is consistent with other pattern-of-care studies across these eras. ${ }^{20}$

Evidence for stage migration in our study includes the improved survival in locally advanced disease (Figure 2B) for those who undergo FDG PET. However, in contrast to the origi- nal report of Feinstein et al, ${ }^{13}$ our study did not find an improved overall survival within the whole cohort or an improved lower stage-specific survival for those who received the newer diagnostic technology (FDG PET) compared with those who did not. The theoretical up-staging of worse-prognosis patients out of the lower-stage population did not appear to improve the survival of patients with localized disease. This may, in part, reflect the fact that certain HNC sites have a high enough probability of occult nodal metastases to warrant elec- 


\begin{tabular}{|c|c|c|}
\hline Characteristics & $\mathrm{HR}(95 \% \mathrm{Cl})$ & $P$ Value \\
\hline \multicolumn{3}{|l|}{ Tumor site } \\
\hline Oral cavity & 1 [Reference] & 1 [Reference] \\
\hline Oropharynx & $0.991(0.507-1.937)$ & .98 \\
\hline Nasopharynx & $1.415(0.562-3.561)$ & .46 \\
\hline $\begin{array}{l}\text { Larynx and/or } \\
\text { hypopharynx }\end{array}$ & $0.987(0.572-1.702)$ & .96 \\
\hline Salivary gland & $1.507(0.796-2.852)$ & .21 \\
\hline $\begin{array}{l}\text { Sinuses and/or } \\
\text { pharynx unspecified }\end{array}$ & $2.826(1.344-5.942)$ & .006 \\
\hline $\begin{array}{l}\text { Age at diagnosis } \\
\text { (continuous) }\end{array}$ & $1.039(1.022-1.057)$ & $<.001$ \\
\hline Female vs male & $0.986(0.653-1.489)$ & .95 \\
\hline $\begin{array}{l}\text { Charlson comorbidity } \\
\text { score vs } 0\end{array}$ & $1.122(0.982-1.281)$ & .09 \\
\hline \multicolumn{3}{|l|}{ Treatment } \\
\hline No treatment & 1 [Reference] & 1 [Reference] \\
\hline Surgery & $1.031(0.681-1.561)$ & .88 \\
\hline Radiotherapy & $1.028(0.633-1.670)$ & .91 \\
\hline Chemotherapy & $1.973(1.268-3.072)$ & .003 \\
\hline PET use vs no PET & $1.208(0.778-1.877)$ & .40 \\
\hline
\end{tabular}

Abbreviations: $\mathrm{HR}$, hazard ratio; $\mathrm{PET}$, positron emission tomography.

a Locally advanced disease is defined as stage III, IVA, or IVB disease $(n=399)$, excluding stage IVC. Localized disease (stage I or II) was used as reference.

tive nodal dissection or irradiation even with a negative FDG PET finding. Thus, there might not be a major difference in the treatment volumes or prognoses of those patients remaining in localized disease population even after FDG PET upstaging. However, the up-staging of patients with nodal disease identified only by FDG PET improved the survival of all patients with nodal disease compared with those with obviously identified (no FDG PET) locally advanced disease.

One of the potential benefits of a staging FDG PET scan is the identification of previously unknown metastatic disease. This could prevent the use of intensive local regional therapies in the setting of previously unknown metastatic disease. The present study found an increase in the number of PET patients who received chemotherapy $(43.1 \%$ vs $17.5 \%)(P<.001)$. However, our study also found that patients who underwent FDG PET were less likely to receive no treatment compared with patients who did not undergo FDG PET. This finding is consistent with prospective studies that showed that few patients have their treatment intent changed to a palliative course because of FDG PET results. ${ }^{9,10}$ One limitation, however, is that we cannot identify which patients did not receive PET because they were already known to have advanced metastatic disease. Once a palliative course is already chosen, there may be no utility to the use of PET.

Although there is a large discrepancy between follow-up times for pre-PET and PET era patients, there was not as large a discrepancy between PET and no-PET patients within the PET era. To address this discrepancy, the interera comparison was limited such that only patients with at least 2 years of follow-up in the PET era were assessed. For the PET vs no-PET analyses, we used patients diagnosed from 2005 through 2008 (PET era only) and used Kaplan-Meier and Cox proportional hazards models, which both account for the differing length of follow-up among sample members.

This study has several limitations. First, inherent in all retrospective studies relying on administrative databases, our study is limited by our inability to evaluate intention of FDG PET scans. Although we limited our definition of pretreatment FDG PET to those who received FDG PET scans 30 days prior to and 60 days after diagnosis, we do not know whether these scans were used for diagnostic, staging, treatment planning, or other reasons. Similarly, the intention of treatment method use is also assumed based on timing of encounters. Second, study cohort members were limited to insured individuals who received their care from 1 of 4 integrated health care systems. These patients may not be representative of patients with cancer at other institutions; thus limiting the generalizability of the findings of this study. Third, this EMR-andencounter-based study was only meant to address the association between FDG PET and up-staging and/or survival. There are many other ways that FDG PET can clinically affect patients, including changing radiation treatment volumes, ${ }^{21,22}$ detecting primary sites for HNC of unknown primary, ${ }^{23}$ and improving surveillance after treatment. ${ }^{24}$ None of these clinical outcomes was addressed in this study.

\section{Conclusions}

Within this cohort of patients with HNC, FDG PET use appeared to be associated with up-staging at diagnosis and improved survival in patients with locally advanced disease but no difference in overall survival for the entire cohort. The improved stage-specific survival is likely a reflection of stage migration. The ability of FDG PET to affect patient management for individual patients remains an important area of future research.

\section{ARTICLE INFORMATION}

Submitted for Publication: December 10, 2013 final revision received March 30, 2014; accepted April 7, 2014.

Published Online: May 29, 2014 doi:10.1001/jamaoto.2014.812.

Author Affiliations: Department of Radiation Oncology, University of North Carolina at Chapel Hill (VanderWalde, Chera); Lineberger Comprehensive Cancer Center, University of North Carolina at Chapel Hill (VanderWalde, Khandani,
Chera); Department of Health Services Policy and Management, Arnold School of Public Health, University of South Carolina, Columbia (Salloum); Department of Health Policy and Management, Gillings School of Public Health, University of North Carolina at Chapel Hill (Liu); The Center for Health Research, Kaiser Permanente Northwest, Portland, Oregon (Hornbrook, O'Keeffe Rosetti); Institute for Health Research, Kaiser Permanente Colorado, Denver (Ritzwoller); Group Health Research Institute, Group Health Cooperative, Seattle, Washington (Fishman); Social and Behavioral
Health and Massey Cancer Center, School of Medicine Virginia Commonwealth University, Richmond (Elston Lafata); Center for Health Policy and Health Services Research, Henry Ford Health System, Detroit, Michigan (Elston Lafata); Division of Nuclear Medicine, Department of Radiology, University of North Carolina at Chapel Hill (Khandani).

Author Contributions: Drs VanderWalde and Chera had full access to all of the data in the study and 
take responsibility for the integrity of the data and the accuracy of the data analysis.

Study concept and design: VanderWalde, Salloum, Liu, Hornbrook, Ritzwoller, Chera.

Acquisition, analysis, or interpretation of data: VanderWalde, Salloum, Liu, Hornbrook, O'Keeffe Rosetti, Ritzwoller, Fishman, Elston Lafata,

Khandani, Chera.

Drafting of the manuscript: VanderWalde, Salloum, Hornbrook, Ritzwoller, Chera.

Critical revision of the manuscript for important intellectual content: VanderWalde, Salloum, Liu, O'Keeffe Rosetti, Fishman, Elston Lafata, Khandani, Chera.

Statistical analysis: VanderWalde, Salloum, Liu, Hornbrook, Ritzwoller, Fishman.

Obtained funding: Fishman.

Administrative, technical, or material support: Hornbrook, O'Keeffe Rosetti, Fishman, Elston Lafata, Khandani.

Study supervision: VanderWalde, Salloum, Fishman, Elston Lafata, Chera.

Conflict of Interest Disclosures: None reported.

Funding/Support: This research was funded in part by National Cancer Institute ( $\mathrm{NCl}$ ) grants R01 CA114204 (Hornbrook), RC2 CA148185 (Ritzwoller), and R25 CA116339 (Salloum) and by NCI Cooperative Agreement U19 CA79689.

Role of the Sponsor: The $\mathrm{NCl}$ had no role in the design and conduct of the study; collection, management, analysis, and interpretation of the data; preparation, review, or approval of the manuscript; and decision to submit the manuscript for publication.

Previous Presentation: This study was presented as a poster discussion presentation at the annual American Society of Clinical Oncology meeting; June 1, 2013; Chicago, Illinois.

Additional Contributions: The following staff members provided some data processing and analysis support for this study: Jenny Staab, PhD, Erin M. Keast, MPH, Erin E. Masterson, MPH, Donald J. Bachman, MS, and Arthur Dixon, MSTM, Kaiser Permanente Northwest, Portland, Oregon; Group Health: Cristi Hanson, BA, Group Health Cooperative, Seattle, Washington; Stephanie Latimer, BS, and Gwyn Saylor, BA, Kaiser Permanente Colorado, Denver; Nonna Akkerman, BS, and Liz Dobie, MPH, Health Alliance Plan-Henry Ford Health System, Detroit, Michigan. No acknowledged person received compensation for their work on this research beyond that provided in the normal course of their employment.

\section{REFERENCES}

1. Laubenbacher C, Saumweber D,

Wagner-Manslau C, et al. Comparison of

fluorine-18-fluorodeoxyglucose PET, MRI, and endoscopy for staging head and neck squamous-cell carcinomas. J Nucl Med. 1995;36 (10):1747-1757.

2. Schwartz DL, Ford E, Rajendran J, et al.

FDG-PET/CT imaging for preradiotherapy staging of head-and-neck squamous cell carcinoma. Int J Radiat Oncol Biol Phys. 2005;61(1):129-136.

3. Ng SH, Yen TC, Chang JT, et al. Prospective study of [18F]fluorodeoxyglucose positron emission tomography and computed tomography and magnetic resonance imaging in oral cavity squamous cell carcinoma with palpably negative neck. J Clin Oncol. 2006;24(27):4371-4376.

4. Dammann F, Horger M, Mueller-Berg M, et al. Rational diagnosis of squamous cell carcinoma of the head and neck region: comparative evaluation of CT, MRI, and 18FDG PET. AJR Am J Roentgenol. 2005:184(4):1326-1331.

5. Zanation AM, Sutton DK, Couch ME, Weissler MC, Shockley WW, Shores CG. Use, accuracy, and implications for patient management of [18F]-2-fluorodeoxyglucose-positron emission/computerized tomography for head and neck tumors. Laryngoscope. 2005;115(7):1186-1190.

6. Daisne JF, Duprez T, Weynand B, et al. Tumor volume in pharyngolaryngeal squamous cell carcinoma: comparison at CT, MR imaging, and FDG PET and validation with surgical specimen. Radiology. 2004:233(1):93-100.

7. Xu G, Li J, Zuo X, Li C. Comparison of whole body positron emission tomography (PET)/PETcomputed tomography and conventional anatomic imaging for detecting distant malignancies in patients with head and neck cancer: a meta-analysis. Laryngoscope. 2012;122(9):19741978.

8. Fleming AJ Jr, Smith SP Jr, Paul CM, et al. Impact of [18F]-2-fluorodeoxyglucose-positron emission tomography/computed tomography on previously untreated head and neck cancer patients. Laryngoscope. 2007;117(7):1173-1179.

9. Lonneux M, Hamoir M, Reychler $\mathrm{H}$, et al. Positron emission tomography with [18F]fluorodeoxyglucose improves staging and patient management in patients with head and neck squamous cell carcinoma: a multicenter prospective study. J Clin Oncol. 2010;28(7):1190-1195.

10. Scott AM, Gunawardana DH, Bartholomeusz D, Ramshaw JE, Lin P. PET changes management and improves prognostic stratification in patients with head and neck cancer: results of a multicenter prospective study. J Nucl Med. 2008;49(10):15931600.

11. Abramyuk A, Appold S, Zöphel K, Baumann M, Abolmaali N. Modification of staging and treatment of head and neck cancer by FDG-PET/CT prior to radiotherapy. Strahlenther Onkol. 2013;189(3):197201.

12. Hillner BE, Tosteson AN, Song Y, et al. Growth in the use of PET for six cancer types after coverage by Medicare: additive or replacement? J Am Coll Radiol. 2012;9(1):33-41.

13. Feinstein AR, Sosin DM, Wells CK. The Will Rogers phenomenon: stage migration and new diagnostic techniques as a source of misleading statistics for survival in cancer. N Engl J Med. 1985; 312(25):1604-1608.

14. Deyo RA, Cherkin DC, Ciol MA. Adapting a clinical comorbidity index for use with ICD-9-CM administrative databases. J Clin Epidemiol. 1992;45 (6):613-619.

15. Smith-Bindman R, Miglioretti DL, Johnson E, et al. Use of diagnostic imaging studies and associated radiation exposure for patients enrolled in large integrated health care systems, 1996-2010. JAMA. 2012;307(22):2400-2409.

16. Dinan MA, Curtis LH, Carpenter WR, et al. Stage migration, selection bias, and survival associated with the adoption of positron emission tomography among Medicare beneficiaries with non-small-cell lung cancer, 1998-2003. J Clin Oncol. 2012;30(22): 2725-2730.

17. Fried D, Khandani A, Shores C, et al. Matched cohort analysis of the effect of pretreatment positron emission tomography on clinical outcomes of patients with head and neck cancer treated with definitive chemoradiotherapy. Head Neck. 2012. 34(3):412-417.

18. Gillison ML, Broutian T, Pickard RK, et al. Prevalence of oral HPV infection in the United States, 2009-2010. JAMA. 2012;307(7):693-703.

19. Ang KK, Harris J, Wheeler $R$, et al. Human papillomavirus and survival of patients with oropharyngeal cancer. N Engl J Med. 2010;363(1): 24-35.

20. VanderWalde NA, Meyer AM, Liu H, et al. Patterns of care in older patients with squamous cell carcinoma of the head and neck: a surveillance, epidemiology, and end results-Medicare analysis. J Geriatr Oncol. 2013;4(3):262-270.

21. Paulino AC, Koshy M, Howell R, Schuster D, Davis LW. Comparison of CT- and FDG-PET-defined gross tumor volume in intensity-modulated radiotherapy for head-and-neck cancer. Int J Radiat Oncol Biol Phys. 2005; 61(5):1385-1392.

22. Garg MK, Glanzman J, Kalnicki S. The evolving role of positron emission tomography-computed tomography in organ-preserving treatment of head and neck cancer. Semin Nucl Med. 2012;42(5):320327.

23. Paul SA, Stoeckli SJ, von Schulthess GK, Goerres GW. FDG PET and PET/CT for the detection of the primary tumour in patients with cervical non-squamous cell carcinoma metastasis of an unknown primary. Eur Arch Otorhinolaryngol. 2007; 264(2):189-195.

24. Kim G, Kim YS, Han EJ, et al. FDG-PET/CT as prognostic factor and surveillance tool for postoperative radiation recurrence in locally advanced head and neck cancer. Radiat Oncol $\mathrm{J}$. 2011;29(4):243-251. 